\title{
SHNOL-TYPE THEOREM FOR THE AGMON GROUND STATE
}

\author{
SIEGFRIED BECKUS, YEHUDA PINCHOVER
}

\begin{abstract}
Let $H$ be a Schrödinger operator defined on a noncompact Riemannian manifold $\Omega$, and let $W \in L^{\infty}(\Omega ; \mathbb{R})$. Suppose that the operator $H+W$ is critical in $\Omega$, and let $\varphi$ be the corresponding Agmon ground state. We prove that if $u$ is a generalized eigenfunction of $H$ satisfying $|u| \leq \varphi$ in $\Omega$, then the corresponding eigenvalue is in the spectrum of $H$. The conclusion also holds true if for some $K \Subset \Omega$ the operator $H$ admits a positive solution in $\tilde{\Omega}=\Omega \backslash K$, and $|u| \leq \psi$ in $\tilde{\Omega}$, where $\psi$ is a positive solution of minimal growth in a neighborhood of infinity in $\Omega$.

Under natural assumptions, this result holds true also in the context of infinite graphs, and Dirichlet forms.
\end{abstract}

2000 Mathematics Subject Classification. Primary 35P05; Secondary 35B09, 35J10, 35R02, 39A12, 81Q10, 81Q35.

Keywords. Caccioppoli inequality, Schrödinger operators, generalized eigenfunction, Green function, ground state, positive solutions, Shnol theorem, weighted graphs.

\section{INTRODUCTION}

In 1957, Shnol [Shn57] proved that if a generalized eigenfunction of a Schrödinger operator $H$ on $\mathbb{R}^{d}$ with potential bounded from below has at most a polynomial growth, then the corresponding energy is in the spectrum of $H$. This celebrated result was independently rediscovered by Simon [Sim81] for a more general class of potentials. Additionally, [Sim81] proved that a dense subset of the spectrum of $H$ admits a polynomial bounded solution. More precisely, it is shown that there is a polynomially bounded solution of the equation $H u=\lambda u$ in $\mathbb{R}^{d}$ for $H$-spectrally a.e. energy $\lambda$, see also [CFKS87, Shu92]. A remarkable generalization of Shnol's theorem in the Dirichlet form setting was proven in [BdMLS09] (see also [HK11, FLW14] and references therein for related results). Also, a converse of Shnol's theorem was proven in the Dirichlet setting [BdMS03]. The proofs rely on a local estimate of the gradient of the generalized eigenfunction, which is needed to control the mixed terms. For this purpose, a Caccioppoli-type inequality is a crucial tool (see [HKM93, BM95], and references therein for the unperturbed operator, and [BdMLS09] for the Dirichlet form setting).

The present work deals with a Shnol-type theorem on a noncompact manifold by replacing the polynomial bound with an object intrinsically defined through the Schrödinger operator. More precisely, let $H$ be a critical Schrödinger operator defined on a noncompact manifold, and denote by $\varphi$ its (Agmon) ground state. It is natural to believe that a Shnol-type theorem is still valid if the generalized eigenfunction is pointwise bounded by $\varphi$. This statement was conjectured by Devyver, Fraas and Pinchover in [DFP14, Conjecture 9.9]. In this work, we provide a positive answer to this conjecture. Let us first provide the class of operators we are mainly interested in, before stating the main theorem. The reader is referred to [Agm83, P07, Pin95] and references therein for a more detailed discussion on criticality theory. 
Let $\Omega$ be a domain in $\mathbb{R}^{d}$ (or a noncompact $d$-dimensional connected Riemannian manifold). Let $m>0$ be a positive measurable function in $\Omega$, and denote $\mathrm{d} m:=m(x) \mathrm{d} x$, where $\mathrm{d} x$ is the volume form of $\Omega$ (which is just the Lebesgue measure in the case of Schrödinger operators on domains of $\left.\mathbb{R}^{n}\right)$. We assume that in any coordinate system $\left(U ; x_{1}, \ldots, x_{d}\right)$ the operator $H$ has the form

$$
H:=-\operatorname{div}(A \nabla)+V,
$$

where the minus divergence is the formal adjoint of the gradient with respect to the measure $m$, and $A$ is a measurable symmetric matrix valued function $A: U \rightarrow \mathbb{R}^{d^{2}}$ satisfying that for every $K \Subset U$ there is a constant $\lambda_{K} \geq 1$ such that $\lambda_{K}^{-1} I \leq A(x) \leq \lambda_{K} I$, where $I$ denotes the $d$-dimensional identity matrix, $A \leq B$ means that $B-A$ is a nonnegative definite matrix, and we use the notation $K \Subset U$ if $K$ is relatively compact in $U$. For $p>d / 2$, let $V \in L_{\text {loc }}^{p}(\Omega, \mathbb{R})$. Define the symmetric form

$$
\mathbf{a}(u, v)=\mathbf{a}_{H}(u, v):=\int_{\Omega}(\langle A \nabla u, \nabla v\rangle+V u \bar{v}) \mathrm{d} m(x)
$$

on $C_{0}^{\infty}(\Omega)$ associated with the Schrödinger operator $H$, where $C_{0}^{\infty}(\Omega)$ denotes the set of compactly supported smooth functions on $\Omega$.

Consider the induced quadratic form which we also denote by $\mathbf{a}=\mathbf{a}_{H}$. We say that $H$ is nonnegative in $\Omega$ (and write $H \geq 0$ in $\Omega$ ) if $\mathbf{a} \geq 0$ on $C_{0}^{\infty}(\Omega)$, and $H$ is semibounded if there is a constant $c \in \mathbb{R}$ such that $\mathbf{a}(v) \geq c\|v\|^{2}$ for all $v \in C_{0}^{\infty}(\Omega)$, where $\|\cdot\|$ is the norm of $L^{2}(\Omega, \mathrm{d} m)$. The operator $H$ is called supercritical in $\Omega$ if a is not nonnegative on $C_{0}^{\infty}(\Omega)$. Suppose that $H \geq 0$ in $\Omega$. Then $H$ is said to be critical in $\Omega$ if for any nonzero nonnegative $W \in L_{\text {loc }}^{p}(\Omega, \mathbb{R})$, with $p>d / 2$, the operator $H-W$ is supercritical in $\Omega$, otherwise, $H$ is subcritical in $\Omega$.

We note that if $H$ is critical in $\Omega$, then (up to a multiplicative constant) the equation $H u=0$ in $\Omega$ admits a unique positive (super)solution. Such a solution is called Agmon ground state (or in short, a ground state), see Theorem 3.3.

It is well-known that if $\mathbf{a}$ is nonnegative on $C_{0}^{\infty}(\Omega)$, then the Dirichlet problem admits a unique solution in every bounded subdomain $\Omega^{\prime} \Subset \Omega[\mathrm{Agm} 83]$. Hence, for $u \in C_{0}^{\infty}(\Omega)$ we have $\mathbf{a}(u)=0$ if and only if $u=0$. In particular, a defines a scalar product on $C_{0}^{\infty}(\Omega)$. Let $\mathcal{H}_{\mathbf{a}}(\Omega)$ be the closure of $C_{0}^{\infty}(\Omega)$ with respect to the norm induced by a. In the sequel it will be evident that the operator $H$ is critical in $\Omega$ if and only if $\mathcal{H}_{\mathbf{a}}(\Omega)$ is not a functional space.

A nonzero function $u \in W_{\text {loc }}^{1,2}(\Omega)$ is called a generalized eigenfunction of $H$ with eigenvalue $\lambda \in \mathbb{R}$ if $\mathbf{a}(u, v)=\lambda \int_{\Omega} u \bar{v} \mathrm{~d} m(x)$ for every $v \in C_{0}^{\infty}(\Omega)$. The main result of this work is the following.

Theorem 1.1. Let $H$ be an operator of the form (1.1), and assume that $H+W$ is critical in $\Omega$ for some real-valued $W \in L^{\infty}(\Omega)$. Denote by $\varphi$ the corresponding ground state of $H+W$. If $u$ is a generalized eigenfunction of $H$ with eigenvalue $\lambda \in \mathbb{R}$ satisfying $|u| \leq \varphi$ in $\Omega$, then $\lambda$ belongs to the $L^{2}(\Omega, \mathrm{d} m)$-spectrum of $H$.

Remark 1.2. In $[\mathrm{P} 07 \mathrm{a}]$ we prove a sufficient condition, in terms of the behavior of a ground state of a critical Schrödinger operator $H_{1}$, such that a positive (sub)solution of a Schrödinger operator $H$ is a ground state. In a certain sense, Theorem 1.1 is a generalization of the main result of $[\mathrm{P} 07 \mathrm{a}]$.

If $H$ is subcritical, or more generally, the equation $H w=0$ admits a positive solution in $\Omega \backslash K$, where $K \Subset \Omega$, then there exists a real-valued $W \in C_{0}(\Omega)$ such that $H+W$ is critical [P07, Pin95], where $C_{0}(\Omega)$ is the space of all continuous compactly supported functions on $\Omega$. Hence, Theorem 1.1 applies in this case. In particular, we have the following result. 
Corollary 1.3. Let $H$ be an operator of the form (1.1), and assume that $H$ is subcritical in $\Omega$. Let $x_{0} \in \Omega$ be a fixed reference point, and denote by $G(x):=G_{H}^{\Omega}\left(x, x_{0}\right)$ the positive minimal Green function of $H$ in $\Omega$ with a pole at $x_{0}$. If $u$ is a generalized eigenfunction of $H$ with eigenvalue $\lambda \in \mathbb{R}$, and satisfies $|u| \leq G$ in $\Omega \backslash B\left(x_{0}, r\right)$ for some $r>0$, then $\lambda$ belongs to the $L^{2}$-spectrum of $H$.

Remark 1.4. If $H$ is supercritical and semibounded, then there exists a constant $c>0$ such that $\tilde{H}:=H+c I \geq 0$ on $C_{0}^{\infty}(\Omega)$. Then either Theorem 1.1 or Corollary 1.3 clearly applies to $\tilde{H}$ and hence to generalized eigenfunctions of $H$.

The proof of Theorem 1.1 hinges on a Weyl-type argument combined with a ground state transform (see Section 3) which eliminates the potential $V$. Let us provide some examples where our main result (Theorem 1.1) applies.

Example 1.5. Let $\Omega \subseteq \mathbb{R}^{d}$ be a domain or a noncompact Riemannian manifold as above. Assume that the operator $H:=-\operatorname{div}(A \nabla)$ on $L^{2}(\Omega, \mathrm{d} m)$ is critical in $\Omega$. The corresponding form acts on $C_{0}^{\infty}(\Omega)$ as

$$
\mathbf{a}(u):=\int_{\Omega}\langle A \nabla u, \nabla u\rangle \mathrm{d} m .
$$

Obviously, the ground state of $H$ in $\Omega$ is the constant function 1. It follows from Theorem 1.1 that if $u_{\lambda}$ is a bounded generalized eigenfunction of $H$ with an eigenvalue $\lambda$, then $\lambda \in \sigma(H)$. We note that particular cases of the present example are clearly given by parabolic Riemannian manifolds or more generally by critical weighted Laplace-Beltrami operators.

The aim of the following example is to provide a simple proof of the well known fact that the spectrum of the Laplacian on $\mathbb{R}^{d}$ is equal to $[0, \infty)$.

Example 1.6. Let $H$ be the Laplacian on $\mathbb{R}$. Then $H$ is critical in $\mathbb{R}$ with a ground state equal 1. Hence, $\sigma(H) \subset[0, \infty)$. Moreover, for any $k \in \mathbb{R}$, the exponential function $\mathrm{e}^{\mathrm{i} k x}$ is a bounded generalized eigenfunction with eigenvalue $\lambda=k^{2}$. Theorem 1.1 implies that $\sigma(H)=[0, \infty)$. In particular, for any $\lambda \geq 0$ there exists a Weyl sequence $\left\{\phi_{i, \lambda}\right\}$ for the operator $H-\lambda$.

Consider now the Laplacian $-\Delta$ on $\mathbb{R}^{d}$, and denote by $x=\left(x_{1}, \ldots, x_{d}\right)$ a point in $\mathbb{R}^{d}$. It can be easily verified that the sequence $\left\{\prod_{j=d}^{n} \phi_{i, \lambda}\left(x_{j}\right)\right\}$ is a Weyl sequence for $-\Delta-d \lambda$ in $\mathbb{R}^{d}$, and hence, the spectrum of the Laplacian on $\mathbb{R}^{d}$ equals to $[0, \infty)$.

Example 1.7. Let $H$ be a symmetric subcritical operator on $\Omega$ of the form (1.1). Let $W \geq 0$ be the optimal Hardy-weight that is given in [DFP14, Theorem 2.2]. Assume further that $W>0$ in $\Omega$. Then the operator $W^{-1} H-1$ is critical in $\Omega$ with a ground state $u_{0}$, and $u_{0} \notin L^{2}(\Omega, W \mathrm{~d} x)$. Moreover, for any $\lambda \geq 1$ the operator $W^{-1} H$ admits generalized eigenfunction $u_{\lambda}$ satisfying $\left|u_{\lambda}\right| \leq u_{0}$ in $\Omega$, see [DFP14, Lemma 7.1 and the proof of Theorem 7.2].

Theorem 1.1 implies that $\sigma\left(W^{-1} H\right)=[1, \infty)$. This gives a simple alternative proof for the last statement of [DFP14, Theorem 2.2].

The outline of the present paper is as follows. In Section 2, we provide a short summary on the theory of forms and in particular, we present a Weyl sequence assertion for forms. Section 3 is devoted to a discussion of the basic notions and results we are using. The Caccioppoli type estimate is proven in Section 4. The proof of the main theorem is then presented in Section 5. Finally, in Section 6 we shortly discuss Dirichlet forms and the possible extension of our result to this setting, where we mainly focus on discrete Schrödinger operators on infinite graphs. 


\section{WEYL-TYPE ARGUMENT}

In the present section we consider a more general situation than considered hitherto. We provide a short summary of the concepts we are using. Let $\Omega$ be a locally compact, separable and connected metric space and $m$ be a positive Radon measure with support $\Omega$. Let $f, g: \Omega \rightarrow$ $(0, \infty)$. We write $f \asymp g$ in $\Omega$ if there exists a positive constant $C$ such that $C^{-1} g \leq f \leq C g$ in $\Omega$.

In order to prove our main result we use a Weyl sequence argument. In the general situation we are dealing with, it is convenient to work with the form associated with the operator. Recall that a form $q: \mathcal{D}(q) \times \mathcal{D}(q) \rightarrow \mathbb{C}$ defined on a linear subspace of a (complex) Hilbert space $\mathcal{H}$ is linear in the first component and complex linear in the second component. Furthermore, $q$ is called symmetric if $q(v, w)=\overline{q(w, v)}$ for all $v, w \in \mathcal{D}(q)$. It is called semibounded if there is a constant $c \in \mathbb{R}$ such that $q(v, v) \geq c\|v\|^{2}$ for all $v \in \mathcal{D}(q)$. If $q$ is a symmetric semibounded form with a constant $c$, then $\|v\|_{q}^{2}:=q(v, v)+(1-c)\|v\|^{2}$ defines a norm on $\mathcal{D}(q)$ satisfying the parallelogram identity. We mainly deal with forms $q$ that are nonnegative $(q \geq 0)$, and hence $q$ is semibounded, where the constant $c$ is equal to zero. The form $q$ is called closed if $\left(\mathcal{D}(q),\|\cdot\|_{q}\right)$ is additionally complete. Every symmetric semibounded form defines a quadratic form by $q(u):=q(u, u)$ for $u \in \mathcal{D}(q)$. For every closed, symmetric, nonnegative form there is a unique self-adjoint operator $H$ associated with $q$. The spectrum of $H$ is denoted by $\sigma(H)$. We denote by $\mathcal{D}_{\text {loc }}(q)$ the local form domain of $q$, see [FLW14].

The following proposition is a Weyl sequence assertion stated for forms. The result is proven in [Sto01, Lemma 1.4.4], see also [BdMLS09, Proposition 2.1]. We provide a proof for the convenience of the reader.

Proposition 2.1 ([Sto01]). Let $q: \mathcal{D}(q) \times \mathcal{D}(q) \rightarrow \mathbb{C}$ be a closed, symmetric, semibounded form, and let $H$ be the associated self-adjoint operator. Then the following assertions are equivalent:

(i) $\lambda \in \sigma(H)$

(ii) There exists a sequence $\left(w_{n}\right)$ in $\mathcal{D}(q)$ with $\lim _{n \rightarrow \infty}\left\|w_{n}\right\|=1$ such that

$$
\lim _{n \rightarrow \infty} \sup _{v \in \mathcal{D}(q),\|v\|_{q} \leq 1}\left|q\left(w_{n}, v\right)-\lambda\left\langle w_{n}, v\right\rangle\right|=0 .
$$

Proof. (i) $\Rightarrow$ (ii): Suppose that $\lambda$ admits a normalized eigenfunction $u$ of $H$, then set $w_{n}:=u$ and (ii) follows. Otherwise, let $\left(\tilde{w}_{n}\right)$ be a (classical) Weyl sequence of the operator $H$ for $\lambda$. In general, $\left(\tilde{w}_{n}\right)$ is not contained in the domain $\mathcal{D}(q)$. However, it is straightforward to find an approximating sequence $\left(w_{n}\right)$ satisfying $(2.1)$ by using that $\mathcal{D}(q)$ is a core of $\mathcal{D}(H)$.

(ii) $\Rightarrow(\mathrm{i})$ : Let $\left(w_{n}\right)$ be a sequence in $\mathcal{D}(q)$ satisfying $\lim _{n \rightarrow \infty}\left\|w_{n}\right\|=1$ and (2.1). Assume, to the contrary, that $\lambda \in \rho(H)$. The boundedness of the resolvent $(H-\lambda)^{-1}$ implies the finiteness of $C:=\sup _{n \in \mathbb{N}}\left\|(H-\lambda)^{-1} w_{n}\right\|_{q}$. Since $q(u, v)=\langle H u, v\rangle$ for $u, v \in D(q)$, we derive with the previous considerations

$$
\left\|w_{n}\right\|^{2}=\left|q\left(w_{n},(H-\lambda)^{-1} w_{n}\right)-\lambda\left\langle w_{n},(H-\lambda)^{-1} w_{n}\right\rangle\right| \leq C \sup _{v \in \mathcal{D}(q),\|v\|_{q} \leq 1}\left|q\left(w_{n}, v\right)-\lambda\left\langle w_{n}, v\right\rangle\right| .
$$

The term on the right hand side tends to zero if $n \rightarrow \infty$, contradicting $\lim _{n \rightarrow \infty}\left\|w_{n}\right\|=1$.

\section{Null-SEquence And the Ground state transform}

Let $\Omega$ be a domain in $\mathbb{R}^{d}$ (or a noncompact $d$-dimensional connected Riemannian manifold). Let $\mathbf{a}:=\mathbf{a}_{H}$ be the form associated with the Schrödinger operator $H=-\operatorname{div}(A \nabla)+V$ in $\Omega$. A 
nonzero function $u \in W_{\text {loc }}^{1,2}(\Omega)$ is called H-harmonic in $\Omega$ if $\mathbf{a}(u, v)=0$ for every $v \in C_{0}^{\infty}(\Omega)$. A function $u \in W_{\text {loc }}^{1,2}(\Omega)$ is called $H$-superharmonic in $\Omega$ if $\mathbf{a}(u, v) \geq 0$ for every nonnegative function $v \in C_{0}^{\infty}(\Omega)$. The cone of all positive $H$-harmonic functions in $\Omega$ is denoted by $\mathcal{C}_{H}(\Omega)$. Next, we introduce the notion of a ground state which is a central object of our considerations.

Definition 3.1. Let $K \Subset \Omega$. A positive $H$-harmonic function $u$ in $\Omega \backslash K$ is called a positive $H$-harmonic of minimal growth in a neighborhood of infinity in $\Omega$ if for every $K \Subset K^{\prime} \Subset \Omega$ with smooth boundary and each positive $H$-superharmonic $v \in C\left(\overline{\Omega \backslash K^{\prime}}\right)$, the inequality $u \leq v$ on the boundary $\partial K^{\prime}$ implies $u \leq v$ in $\Omega \backslash K^{\prime}$. A positive solution $u \in \mathcal{C}_{H}(\Omega)$ that has minimal growth at infinity in $\Omega$ is called a ground state of $H$ in $\Omega$.

Definition 3.2. Let $H$ be an operator of the form (1.1), and assume that the quadratic form $\mathbf{a}:=\mathbf{a}_{H}$ is nonnegative on $C_{0}^{\infty}(\Omega)$. A sequence $\left(\varphi_{n}\right) \subset C_{0}^{\infty}(\Omega)$ of nonnegative functions is called a null-sequence for $\mathbf{a}$ if there exists a ball $B \Subset \Omega$ such that $\int_{B} \varphi_{n}^{2} \mathrm{~d} m=1$ and $\lim _{n \rightarrow \infty} \mathbf{a}\left(\varphi_{n}\right)=0$. Furthermore, a positive function $\varphi \in \mathcal{D}_{\text {loc }}$ is called a null-state of $\mathbf{a}$ if there exists a null-sequence $\left(\varphi_{n}\right)$ such that $\left(\varphi_{n}\right)$ converges in $L_{\text {loc }}^{2}(\Omega)$ to $\varphi$.

The following characterization of criticality is well known in various contexts, we refer to the following papers [P07, PT06, Pin95, KPP16] and references therein.

Theorem 3.3 (Criticality characterization). Let $H$ be an operator of the form (1.1), and assume that the quadratic form $\mathbf{a}_{H}$ is nonnegative on $C_{0}^{\infty}(\Omega)$. Then, the following assertions are equivalent:

(i) $H$ is critical in $\Omega$.

(ii) $H$ admits a ground state in $\Omega$.

(ii) $H$ does not admit a positive minimal Green function in $\Omega$.

(iii) $H$ admits a unique (up to a multiplicative constant) positive $H$-superharmonic function in $\Omega$.

(iv) For any open ball $B \Subset \Omega$, there is a null-sequence $\left(\varphi_{n}\right)$ such that $\int_{B} \varphi_{n}(x)^{2} \mathrm{~d} m=1$ for all $n \geq 0$.

(v) There exists a null-sequence $\left(\varphi_{n}\right)$ satisfying $0 \leq \varphi_{n} \leq v$ in $\Omega$, where $v$ is a positive $H$-harmonic function on $\Omega$, and $\varphi_{n}(x) \rightarrow v(x)$ locally uniformly in $\Omega$.

In particular, $\varphi$ is a null-state if and only if it is a ground state.

Note that $\operatorname{dim}\left(\mathcal{C}_{H}(\Omega)\right)=1$ holds whenever $H$ is critical. Thus, a ground state is unique (up to a multiplicative constant).

Another important ingredient is the ground state transform. For a positive continuous function $h \in W_{\text {loc }}^{1,2}(\Omega) \cap C(\Omega)$, define

$$
T_{h}(v):=\frac{v}{h} .
$$

Note that $T_{h}^{-1}=T_{h^{-1}}$ holds. The operator

$$
H_{h}:=T_{h} \circ H \circ T_{h}^{-1}
$$

is called the $h$-transform of $H$. If $\varphi \in \mathcal{C}_{H}(\Omega)$, then $H_{\varphi}$ is called a ground state transform of $H$. Furthermore, $\left.T_{\varphi}\right|_{\mathcal{D}_{\text {loc }} \cap L^{2}(\Omega)}$ extends to an isometry between $L^{2}(\Omega, \mathrm{d} m)$ and $L^{2}\left(\Omega, \varphi^{2} \mathrm{~d} m\right)$, see for example [DFP14, Proposition 4.15]. Hence, $\sigma(H)=\sigma\left(H_{\varphi}\right)$ follows. The reader is referred to [DFP14, KPP16] and references therein for further background on the ground state transform. Consider the Schrödinger operator $H=-\operatorname{div}(A \nabla)+V$ on $\Omega$ with associated nonnegative form

$$
\mathbf{a}(u, v):=\int_{\Omega}(\langle A \nabla u, \nabla v\rangle+V u \bar{v}) \mathrm{d} m .
$$


Suppose that $H$ is critical in $\Omega$ with ground state $\varphi$. Then the quadratic form $\mathbf{a}_{\varphi}$ of the operator $H_{\varphi}$ is given by

$$
\mathbf{a}_{\varphi}(u, v):=\int_{\Omega}\langle A \nabla u, \nabla v\rangle \mathrm{d} \mu(x)
$$

where the measure $\mu$ is given by $\varphi^{2} \mathrm{~d} m$. For more details see for example [DFP14]. It is worth mentioning that $H_{\varphi}$ is critical in $\Omega$, and its ground state is given by the constant function 1. This fact will be intensively used. Finally, let us point out that we obtain the following Cauchy-Schwarz inequality

$$
\begin{aligned}
\left|\int_{\Omega} u v\langle A \nabla v, \nabla u\rangle \mathrm{d} \mu\right| & \leq \int_{\Omega}|u||\langle A \nabla v, \nabla v\rangle|^{\frac{1}{2}}|v||\langle A \nabla u, \nabla u\rangle|^{\frac{1}{2}} \mathrm{~d} \mu \\
& \leq\left(\int_{\Omega}|u|^{2}\langle A \nabla v, \nabla v\rangle \mathrm{d} \mu\right)^{\frac{1}{2}}\left(\int_{\Omega}|v|^{2}\langle A \nabla u, \nabla u\rangle \mathrm{d} \mu\right)^{\frac{1}{2}}
\end{aligned}
$$

by using that $A$ is nonnegative definite.

\section{Caccioppoli-type estimate}

Throughout this section we consider a nonnegative form a given by

$$
\mathbf{a}(u, v):=\int_{\Omega}\langle A \nabla u, \nabla v\rangle d \mu(x),
$$

with the associated self-adjoint operator $L:=-\operatorname{div}(A \nabla)$ on $L^{2}(\Omega, \mathrm{d} \mu)$. For a real-valued potential $W \in L^{\infty}(\Omega)$, consider the self-adjoint operator $L+W:=-\operatorname{div}(A \nabla)+W$ on $L^{2}(\Omega, \mathrm{d} \mu)$. The associated symmetric form $q$ of $L+W$ is defined by $q(u, v)=\mathbf{a}(u, v)+\langle W u, v\rangle$, where $\langle\cdot, \cdot\rangle$ and $\|\cdot\|$ are the inner product and the norm on $L^{2}(\Omega, \mathrm{d} \mu)$. Clearly, $q$ is semibounded with constant $c:=-\|W\|_{\infty}$ as a is nonnegative. Thus, $\|\cdot\|_{q}$ is defined by $\|v\|_{q}^{2}:=q(v, v)+\left(1+\|W\|_{\infty}\right)\|v\|^{2}$ (see Section 2). We have the following Caccioppoli-type estimate.

Proposition 4.1. Let $W \in L^{\infty}(\Omega)$ be real-valued, and let $u \in W_{\mathrm{loc}}^{1,2}(\Omega)$ be a generalized eigenfunction of the operator $L+W$ with eigenvalue $\lambda \in \mathbb{R}$ satisfying $|u| \leq 1$ in $\Omega$. Then

$$
\int_{\Omega}|v|^{2}\langle A \nabla u, \nabla u\rangle \mathrm{d} \mu \leq\left(1+\sqrt{1+|\lambda|+\|W\|_{\infty}}\right)^{2}
$$

holds for every $v \in \mathcal{D}(q)$ satisfying $\|v\|_{q} \leq 1$.

Proof. We first show the desired estimate for $v \in C_{0}^{\infty}(\Omega)$ with $\|v\|_{q} \leq 1$. Set

$$
z:=\sqrt{\int_{\Omega}|v|^{2}\langle A \nabla u, \nabla u\rangle \mathrm{d} \mu} .
$$

The constraint $\|v\|_{q} \leq 1$ implies $\|v\| \leq 1$ and $\mathbf{a}(v, v) \leq 1$ since $\langle W v, v\rangle+\|W\|_{\infty}\|v\|^{2} \geq 0$. Thus, a short computation yields

$$
\begin{aligned}
z^{2} & =\left|\int_{\Omega}\langle A \nabla(\bar{v} v u), \nabla u\rangle \mathrm{d} \mu-\int_{\Omega} u\langle A \nabla(\bar{v} v), \nabla u\rangle \mathrm{d} \mu\right| \\
& \leq\left.\left|\int_{\Omega}(\lambda-W)\right| v\right|^{2} \underbrace{|u|^{2}}_{\leq 1} \mathrm{~d} \mu|+| \int_{\Omega} u \bar{v}\langle A \nabla v, \nabla u\rangle \mathrm{d} \mu|+| \int_{\Omega} u v\langle A \nabla \bar{v}, \nabla u\rangle \mathrm{d} \mu \mid
\end{aligned}
$$




$$
\begin{aligned}
& \stackrel{\text { C.S. }}{\leq}\left(|\lambda|+\|W\|_{\infty}\right) \underbrace{\|v\|^{2}}_{\leq 1}+2 \underbrace{\left(\int_{\Omega}|u|^{2}\langle A \nabla v, \nabla v\rangle \mathrm{d} \mu\right)^{\frac{1}{2}}}_{\leq \sqrt{\mathbf{a}(v, v)} \leq 1}\left(\int_{\Omega}|v|^{2}\langle A \nabla u, \nabla u\rangle \mathrm{d} \mu\right)^{\frac{1}{2}} \\
& \leq|\lambda|+\|W\|_{\infty}+2 z .
\end{aligned}
$$

Since $v$ is compactly supported, the above integrals are all well-defined. An elementary computation implies $0 \leq z \leq 1+\sqrt{1+|\lambda|+\|W\|_{\infty}}$ showing the desired estimate for $v \in C_{0}^{\infty}(\Omega)$ with $\|v\|_{q} \leq 1$.

Now consider $v \in \mathcal{D}(q)$ satisfying $\|v\|_{q} \leq 1$. Since $C_{0}^{\infty}(\Omega)$ is dense in $\mathcal{D}(q)$ with respect to $\|\cdot\|_{q}$, there exists a sequence $\left(v_{n}\right) \subseteq C_{0}^{\infty}(\Omega)$ such that $\lim _{n \rightarrow \infty}\left\|v-v_{n}\right\|_{q}=0$ and $\left\|v_{n}\right\|_{q} \leq 1$. Thus, $v-v_{n}$ tends to zero in the $L^{2}$-norm and so there is no loss of generality in assuming that $\left(v_{n}\right)$ converges $\mathrm{d} \mu$-a.e. to $v$. Applying Fatou's Lemma, we get

$$
\int_{\Omega}|v|^{2}\langle A \nabla u, \nabla u\rangle \mathrm{d} \mu \leq \liminf _{n \rightarrow \infty} \int_{\Omega}\left|v_{n}\right|^{2}\langle A \nabla u, \nabla u\rangle \mathrm{d} \mu \leq\left(1+\sqrt{1+|\lambda|+\|W\|_{\infty}}\right)^{2} .
$$

Thus, the proposition is proved.

\section{SHNOL-TYPE THEOREM}

Throughout this section we consider a nonnegative form a given by

$$
\mathbf{a}(u, v):=\int_{\Omega}\langle A \nabla u, \nabla v\rangle \mathrm{d} \mu(x),
$$

with associated self-adjoint operator $L:=-\operatorname{div}(A \nabla)$ on $L^{2}(\Omega, \mathrm{d} \mu)$. Like in the previous section, $q$ denotes the form associated with $L+W$ for a real-valued potential $W \in L^{\infty}(\Omega)$.

Proposition 5.1. Suppose that $L$ is critical in $\Omega$ with the ground state 1 . Let $W \in L^{\infty}(\Omega)$ be real-valued and $u$ be a generalized eigenfunction $L+W$ with eigenvalue $\lambda \in \mathbb{R}$. If $|u| \leq 1$ in $\Omega$, then $\lambda \in \sigma(L+W)$.

Proof. Invoking Theorem 3.3, there exists a null-sequence $\left(\varphi_{n}\right)$ of $L$ satisfying $0 \leq \varphi_{n} \leq 1$ such that $\left(\varphi_{n}\right)$ converges, locally uniformly, to the ground state 1 . If $u \in L^{2}(\Omega, \mathrm{d} \mu)$, then obviously, $\lambda \in \sigma(L+W)$.

So, we may suppose that $u \notin L^{2}(\Omega, \mathrm{d} \mu)$. Thus, $\lim _{n \rightarrow \infty}\left\|\varphi_{n} u\right\|=\infty$ follows by Fatou's Lemma since $\varphi_{n} u$ converges locally to $u$. Hence, there is an $n_{0} \in \mathbb{N}$ such that $\left\|\varphi_{n} u\right\| \geq 1$ for $n \geq n_{0}$. Define $w_{n}:=\frac{\varphi_{n} u}{\left\|\varphi_{n} u\right\|}, n \geq n_{0}$, so, $\left\|w_{n}\right\|=1$.

Denote by $q$ the symmetric semibounded form associated with $L+W$. Let $v \in \mathcal{D}(q)$ be so that $\|v\|_{q} \leq 1$. Since $u$ is a generalized eigenfunction of $L+W$, we have

$$
\lambda\left\langle w_{n}, v\right\rangle=\frac{\lambda}{\left\|\varphi_{n} u\right\|}\left\langle u, \varphi_{n} v\right\rangle=\frac{1}{\left\|\varphi_{n} u\right\|} q\left(u, \varphi_{n} v\right)=\frac{1}{\left\|\varphi_{n} u\right\|} \mathbf{a}\left(u, \varphi_{n} v\right)+\left\langle W w_{n}, v\right\rangle .
$$

Hence, $\left|q\left(w_{n}, v\right)-\lambda\left\langle w_{n}, v\right\rangle\right|=\frac{1}{\left\|\varphi_{n} u\right\|}\left|\mathbf{a}\left(\varphi_{n} u, v\right)-\mathbf{a}\left(u, \varphi_{n} v\right)\right|$. Therefore, the Leibniz rule implies

$$
\left|q\left(w_{n}, v\right)-\lambda\left\langle w_{n}, v\right\rangle\right|=\frac{1}{\left\|\varphi_{n} u\right\|}\left|\int_{\Omega} u 1\left\langle A \nabla \varphi_{n}, \nabla v\right\rangle \mathrm{d} \mu-\int_{\Omega} \bar{v} 1\left\langle A \nabla u, \nabla \varphi_{n}\right\rangle \mathrm{d} \mu\right| .
$$

Applying the triangle and the Cauchy-Schwarz inequalities, the previous terms are estimated by

$$
\frac{1}{\left\|\varphi_{n} u\right\|}\left[\left(\int_{\Omega}|u|^{2}\left\langle A \nabla \varphi_{n}, \nabla \varphi_{n}\right\rangle \mathrm{d} \mu\right)^{\frac{1}{2}} \mathbf{a}(v, v)^{\frac{1}{2}}+\left(\int_{\Omega}|v|^{2}\langle A \nabla u, \nabla u\rangle \mathrm{d} \mu\right)^{\frac{1}{2}} \mathbf{a}\left(\varphi_{n}, \varphi_{n}\right)^{\frac{1}{2}}\right]
$$




$$
\leq \frac{\mathbf{a}\left(\varphi_{n}, \varphi_{n}\right)^{\frac{1}{2}}}{\left\|\varphi_{n} u\right\|}\left(1+\left(\int_{\Omega}|v|^{2}\langle A \nabla u, \nabla u\rangle \mathrm{d} \mu\right)^{\frac{1}{2}}\right),
$$

where $|u| \leq 1$ and $\|v\|_{q} \leq 1$ is used to obtain the second line.

Due to Proposition 4.1, the integral $\int_{\Omega}|v|^{2}\langle A \nabla u, \nabla u\rangle \mathrm{d} \mu$ is bounded by a constant (depending on $\|W\|_{\infty}$ and $\lambda$ ), and in particular, it is well-defined. Since $\left\|\varphi_{n} u\right\| \geq 1$ for $n \geq n_{0}$, we deduce

$$
\left|q\left(w_{n}, v\right)-\lambda\left\langle w_{n}, v\right\rangle\right| \leq\left(2+\sqrt{1+|\lambda|+\|W\|_{\infty}}\right) \mathbf{a}\left(\varphi_{n}, \varphi_{n}\right)^{\frac{1}{2}},
$$

for all $n \geq n_{0}$ and $v \in \mathcal{D}(q)$ with $\|v\|_{q} \leq 1$. Since $\left(\varphi_{n}\right)$ is a null-sequence of $L$, the previous estimate implies that $\left|q\left(w_{n}, v\right)-\lambda\left\langle w_{n}, v\right\rangle\right|$ tends to zero uniformly in $v \in \mathcal{D}(q)$ with $\|v\|_{q} \leq 1$. Additionally, $q$ is a closed, symmetric, semibounded form since $\mathbf{a}$ is nonnegative and $W \in L^{\infty}(\Omega)$ is real-valued. Consequently, $\lambda \in \sigma(L+W)$ follows by Proposition 2.1.

Now, the main theorem is a direct consequence of the previous considerations.

Proof.[Proof of Theorem 1.1] Since $H+W$ is critical, Theorem 3.3 implies that $H+W$ admits a ground state $\varphi$. Using the ground state transform $T_{\varphi}$, the operators $L-W$ and $H$ are unitary equivalent where $L:=T_{\varphi} \circ(H+W) \circ T_{\varphi}^{-1}$. Furthermore, $T_{\varphi} \varphi=1$ is the ground state of the critical operator $L$. Set $u_{\varphi}=T_{\varphi} u$. Then $u_{\varphi}$ is a generalized eigenfunction of $L-W$ with eigenvalue $\lambda \in \mathbb{R}$ and $\left|u_{\varphi}\right| \leq 1$. Applying Proposition 5.1 to $L$ with generalized eigenfunction $u_{\varphi}$ of $L-W$ leads to $\lambda \in \sigma(L-W)=\sigma(H)$.

Proof.[Proof of Corollary 1.3] According to [P88, Theorem 4.6] there is a nonnegative $W \in$ $C_{0}(\Omega)$ such that $H-W$ is critical in $\Omega$, and let $\varphi$ be the corresponding ground state. Since $G \asymp \varphi$ [P88, Corollary 4.3] in $\Omega \backslash B\left(x_{0}, r\right)$, it follows that $|u| \leq C \varphi$ in $\Omega$ for some $C>0$. Hence, the corollary follows from Theorem 1.1 applied to the generalized eigenfunction $u / C$ of $H$.

\section{DiRICHLET FORMS}

In the following, we provide a short summary on Dirichlet forms. The more interested reader is referred to [Fuk80, BH91, MR92, FOT94, Dav95] for further background on Dirichlet forms.

Let $X$ be a locally compact, connected, separable metric space with positive measure $m$. Consider a dense subspace $\mathcal{D} \subseteq L^{2}(X, m)$ and a sesquilinear, nonnegative, symmetric map $\mathcal{E}: \mathcal{D} \times \mathcal{D} \rightarrow \mathbb{R}$. If $\mathcal{D}$ is closed in the energy norm $\|\cdot\|_{\mathcal{E}}^{2}:=\mathcal{E}(\cdot, \cdot)+\|\cdot\|^{2}$, then $\mathcal{E}$ is called a closed (symmetric) form in $L^{2}(X, m)$, where $\|\cdot\|$ denotes the $L^{2}$-norm. We associate to $\mathcal{E}$ the unique self-adjoint operator $(H, D(H))$ satisfying $D(H) \subseteq \mathcal{D}$ and $\mathcal{E}(\psi, u)=\langle H \psi, u\rangle$ for all $\psi \in D(H)$ and $u \in \mathcal{D}$. We use the short notation $\mathcal{E}(u)=\mathcal{E}(u, u)$ for $u \in \mathcal{D}$.

The (symmetric) closed form $\mathcal{E}$ is called (symmetric) Dirichlet form whenever $T \circ u \in \mathcal{D}$ and $\mathcal{E}(T \circ u) \leq \mathcal{E}(u)$ holds for all $u \in \mathcal{D}$ and every normal contraction $T: \mathbb{R} \rightarrow \mathbb{R}$. Recall that $T: \mathbb{R} \rightarrow \mathbb{R}$ is called normal contraction if $T(0)=0$ and $|T(x)-T(y)| \leq|x-y|$ for each $x, y \in \mathbb{R}$. A Dirichlet form is called regular if $\mathcal{D} \cap C_{0}(X)$ is dense in $\left(\mathcal{D},\|\cdot\|_{\mathcal{E}}\right)$ and in $\left(C_{0}(X),\|\cdot\|_{\infty}\right)$. Furthermore, a Dirichlet form is called strongly local if $\mathcal{E}(u, v)=0$ whenever $u \in \mathcal{D}$ is constant a.s. on the support of $v \in \mathcal{D}$.

The Beurling-Deny formula asserts that for every symmetric regular Dirichlet form $\mathcal{E}$ there exists a unique decomposition

$$
\mathcal{E}(u, v)=\mathcal{E}^{(a)}(u, v)+\mathcal{E}^{(b)}(u, v)+\mathcal{E}^{(c)}(u, v) \quad u, v \in \mathcal{D},
$$


where $\mathcal{E}^{(a)}$ is the killing term, $\mathcal{E}^{(b)}$ is the jump part and $\mathcal{E}^{(c)}$ is the strongly local part. Each of this parts can be represented as

$$
\mathcal{E}^{(*)}(u, v)=\int_{X} \mathrm{~d} \mu^{(*)}(u, v) \quad * \in\{a, b, c\},
$$

for a suitable Radon measure $\mu^{(*)}$. For $* \in\{a, b, c\}$, the measure $\mu^{(*)}$ satisfies the CauchySchwarz inequality

$$
\left|\int_{X} \psi \varphi \mathrm{d} \mu^{(*)}(u, v)\right| \leq\left(\int_{X}|\psi|^{2} \mathrm{~d} \mu^{(*)}(u, u)\right)^{\frac{1}{2}}\left(\int_{X}|\varphi|^{2} \mathrm{~d} \mu^{(*)}(v, v)\right)^{\frac{1}{2}} .
$$

Given a regular Dirichlet form, it extends to its local form domain, see [FLW14, Section 3.1] for details. An element $u$ in its local domain is called a generalized eigenfunction with eigenvalue $\lambda \in \mathbb{R}$ if $\mathcal{E}(u, v)=\lambda\langle u, v\rangle$ for all $v \in \mathcal{D} \cap C_{0}(X)$.

In order to generalize Proposition 4.1 and Proposition 5.1 to regular Dirichlet forms, one needs additionally an integrated version of the Leibniz rule [FLW14, Theorem 3.7] and a suitable notion of a ground state and null-sequence. The ground state transform is established for a class of strictly local regular Dirichlet forms in [LSV09, LSV11]. Additionally, the authors show the existence of a weak positive solution (Allegretto-Piepenbrink-type theorem) by approximating such a solution under suitable assumptions on the Dirichlet form. In the following, we focus on regular Dirichlet forms over a discrete set $X$ since the existence of a ground state with corresponding null-sequence is known under reasonable assumption, see [KL12, KPP16]. The statements of Theorem 3.3 stay valid in this setting while some estimates need to be adjusted since the chain rule does not hold for discrete Laplacians, see [KPP16].

Let $X$ be a countable infinite set equipped with the discrete topology, and $m: X \rightarrow(0, \infty)$ be a measure on $X$ with full support. Then $\ell^{2}(X, m)$ is defined by all $u: X \rightarrow \mathbb{R}$ satisfying $\sum_{x \in X} u(x)^{2} m(x)<\infty$ equipped with the standard scalar product weighted by $m$. A symmetric weighted graph over $X$ is a map $b: X \times X \rightarrow[0, \infty)$ satisfying $b(x, x)=0, x \in X, b(x, y)=$ $b(y, x), x, y \in X$ and $\sum_{y \in X} b(x, y)<\infty$ for all $x \in X$. We say that $x, y \in X$ are adjacent or neighbors or connected by an edge if $b(x, y)>0$. A graph $b$ is called connected if there is a path connecting every two vertices $x, y \in X$. According to [KL12, Theorem 7$]$, there exists for every regular Dirichlet form $Q$ on $\ell^{2}(X, m)$ a graph $b$ on $X$ with nonnegative $c: X \rightarrow[0, \infty]$ such that $Q=Q_{b, c}$, where

$$
Q_{b, c}(u, v):=\sum_{x, y \in X} b(x, y)(u(x)-u(y))(v(x)-v(y))+\sum_{x \in X} u(x) v(x) c(x)
$$

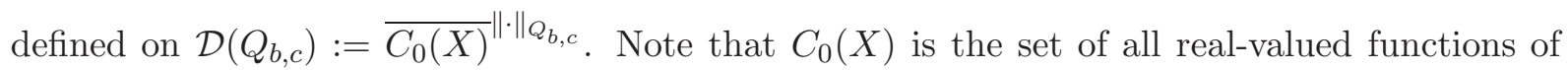
$X$ with finite support. Denote by $H$ the unique operator associated with $Q$. The integrated version of the Leibniz rule reads as follows

$$
\sum_{x, y \in X} d_{b}(u v)(x, y) d_{b} w(x, y)=\sum_{x, y \in X} u(x) d_{b} v(x, y) d_{b}(w)(x, y)+\sum_{x, y \in X} v(x) d_{b} u(x, y) d_{b}(w)(x, y)
$$

where $d_{b} u(x, y):=\sqrt{b(x, y)}(u(x)-u(y))$, see [FLW14, Theorem 3.9] or [HKMW13, Lemma 3.2]. Let $F(X)$ be the set of all $u: X \rightarrow \mathbb{R}$ satisfying $\sum_{y \in X} b(x, y)|f(y)|<\infty$ for each $x \in X$. A function $u \in F(X)$ is called harmonic if $H u=0$ in $X$. Let $Q \geq 0$ on $C_{0}(X)$. Every function $W: X \rightarrow \mathbb{R}$ defines a quadratic form $Q_{W}$ on $C_{0}(X)$ by $Q_{W}(u):=\sum_{x \in X} W(x) u(x)^{2}$. Analogously to the continuous setting, $Q \geq 0$ on $C_{0}(X)$ is said to be subcritical in $X$ if there is 
a nonzero $W: X \rightarrow[0, \infty)$ such that $Q-Q_{W} \geq 0$ on $C_{0}(X)$. Furthermore, a sequence $\left(\varphi_{n}\right)$ of nonnegative functions in $C_{0}(X)$ is called null-sequence if there is an $o \in X$ and a constant $C>0$ such that $\varphi_{n}(o)=C$ for each $n \in \mathbb{N}$ and $Q\left(\varphi_{n}\right) \rightarrow 0$. Note that for a nonnegative quadratic form $Q$ the existence of a positive harmonic function is guaranteed in the case of locally finite graphs [HK11], or in the critical case [KPP16]. In the following, the space of bounded real-valued functions on $X$ equipped with the uniform norm is denoted by $B(X, \mathbb{R})$. With this at hand, we can show the discrete analog of Proposition 4.1 and Proposition 5.1.

Let $Q:=Q_{b, 0}$ be a regular Dirichlet form on $\ell^{2}(X, m)$ with zero potential $c \equiv 0$ and $L$ the associated self-adjoint operator. For $W \in B(X, \mathbb{R})$, the form associated with $L+W$ is denotes by $Q_{b, W}=Q+Q_{W}$ which is semibounded with constant $c:=-\|W\|_{\infty}$ as $Q$ is nonnegative. Let $\|v\|_{Q_{b, W}}:=Q(v, v)+\left(1+\|W\|_{\infty}\right)\|v\|^{2}$. We have the following Caccioppoli-type estimate.

Proposition 6.1. Suppose that $Q:=Q_{b, 0}$ is a regular Dirichlet form on $\ell^{2}(X, m)$ with zero potential $c \equiv 0$ and $L$ the associated self-adjoint operator. For $W \in B(X, \mathbb{R})$, consider a generalized eigenfunction $u \in F(X)$ of $L+W$ with eigenvalue $\lambda \in \mathbb{R}$, such that $|u| \leq 1$ in $X$. Then

$$
\sum_{x, y \in X} v(x)^{2} d_{b} u(x, y)^{2} \leq\left(1+\sqrt{1+|\lambda|+\|W\|_{\infty}}\right)^{2}
$$

holds for every $v \in \mathcal{D}\left(Q_{b, W}\right)$ satisfying $\|v\|_{Q_{b, W}} \leq 1$.

Proof. Let $v \in C_{0}(X)$ be such that $\|v\|_{Q_{b, W}} \leq 1$. A short computation and the symmetry $b(x, y)=b(y, x)$ leads to

$$
\sum_{x, y \in X}\left|d_{b} v^{2}(x, y)\right|\left|d_{b} u(x, y)\right| \leq 2 \sum_{x, y \in X}|v(x)|\left|d_{b} v(x, y)\right|\left|d_{b} u(x, y)\right|,
$$

where each of the sums is finite as $v$ has finite support. Set

$$
z:=\sqrt{\sum_{x, y \in X} v(x)^{2} d_{b} u(x, y)^{2}} .
$$

Like in Proposition 4.1, $\|v\|_{Q_{b, W}} \leq 1$ leads to $\|v\|^{2} \leq 1$ and $Q(v, v) \leq 1$ since $\langle W v, v\rangle+$ $\|W\|_{\infty}\|v\|^{2} \geq 0$. Using that $u$ is a generalized eigenfunction and the Leibniz rule, the estimate

$$
z^{2}=\left|Q\left(u, v^{2} u\right)-\sum_{x, y \in X} u(x) d_{b} v^{2}(x, y) d_{b} u(x, y)\right| \leq|\lambda|+\|W\|_{\infty}+\sum_{x, y \in X}\left|d_{b} v^{2}(x, y)\right|\left|d_{b} u(x, y)\right|
$$

follows by using $|u| \leq 1$ and $\|v\|_{Q_{b, W}} \leq 1$. Hence, $z^{2} \leq|\lambda|+\|W\|_{\infty}+2 z$ is concluded using (6.1) and the Cauchy-Schwarz inequality. Following the lines of the proof of Proposition 4.1 the desired result is derived.

Proposition 6.2. Suppose $Q:=Q_{b, 0}$ is a regular Dirichlet form on $\ell^{2}(X, m)$ with zero potential $c \equiv 0$ such that the associated self-adjoint operator $L$ is critical with ground state 1 . Let $W \in$ $B(X, \mathbb{R})$ and $u \in F(X)$ be a generalized eigenfunction of $L+W$ with eigenvalue $\lambda \in \mathbb{R}$. If $|u| \leq 1$, then $\lambda \in \sigma(L+W)$.

Proof. According to [KPP16, Theorem 2.20], there exists a null-sequence $\left(\varphi_{n}\right)$ of $Q$ satisfying $0 \leq \varphi_{n} \leq 1$ such that $\varphi_{n}(x) \rightarrow 1$ holds for each $x \in X$. We may suppose that $u \notin L^{2}(X, m)$ since otherwise $\lambda \in \sigma(L+W)$ follows immediately. Define the sequence $w_{n}:=\frac{\varphi_{n} u}{\left\|\varphi_{n} u\right\|}, n \in \mathbb{N}$. 
The form associated with $L+W$ is denoted by $Q_{b, W}=Q+Q_{W}$. Using the integrated version of the Leibniz rule and that $u$ is a generalized eigenfunction of $L+W$, we have

$$
\begin{aligned}
&\left|Q_{b, W}\left(w_{n}, v\right)-\lambda\left\langle w_{n}, v\right\rangle\right| \\
&=\frac{1}{\left\|\varphi_{n} u\right\|}\left|\sum_{x, y \in X} u(x) 1 d_{b} \varphi_{n}(x, y) d_{b} v(x, y)-\sum_{x, y \in X} v(x) 1 d_{b} u(x, y) d_{b} \varphi_{n}(x, y)\right|
\end{aligned}
$$

Following the lines of the proof of Proposition 5.1 and using Cauchy-Schwarz, we get

$$
\left|Q_{b, W}\left(w_{n}, v\right)-\lambda\left\langle w_{n}, v\right\rangle\right| \leq \frac{Q\left(\varphi_{n}, \varphi_{n}\right)^{\frac{1}{2}}}{\left\|\varphi_{n} u\right\|}\left(1+\left(\sum_{x, y \in X} v(x)^{2} d_{b} u(x, y)^{2}\right)^{\frac{1}{2}}\right)
$$

for each $v \in \mathcal{D}\left(Q_{b, W}\right)$ satisfying $\|v\|_{Q_{b, W}} \leq 1$. Due to Proposition $6.1, \sum_{x, y \in X} v(x)^{2} d_{b} u(x, y)^{2}$ is bounded by a constant (only depending on $\|W\|_{\infty}$ and $\lambda$ ). Furthermore, there exists an $n_{0} \in \mathbb{N}$ such that $\left\|\varphi_{n} u\right\| \geq 1$ for $n \geq n_{0}$. With this at hand, the previous considerations lead to

$$
\lim _{n \rightarrow \infty} \sup _{v \in \mathcal{D}\left(Q_{b, W}\right),\|v\|_{Q_{b, W}} \leq 1}\left|Q_{b, W}\left(w_{n}, v\right)-\lambda\left\langle w_{n}, v\right\rangle\right|=0
$$

as $\left(\varphi_{n}\right)$ is a null-sequence of $Q$. Thus, Proposition 2.1 yields $\lambda \in \sigma(L+W)$.

Theorem 6.3. Let $b$ be a connected graph, $c: X \rightarrow \mathbb{R}$ and $Q=Q_{b, c}$ be a regular Dirichlet form on $X$ with associated self-adjoint operator $H$. Suppose that there is $a W \in C_{b}(X)$ such that $H+W$ is critical in $X$ with ground state $\varphi$. If $u \in F(X)$ is a generalized eigenfunction of $H$ with eigenvalue $\lambda \in \mathbb{R}$ and $|u| \leq \varphi$ in $X$, then $\lambda \in \sigma(H)$.

Proof. The ground state transform $T_{\varphi}$ eliminates the zero-order term $c$ and $T_{\varphi} \circ(H+W) \circ T_{\varphi}^{-1}$ is critical with ground state 1 , see [KPP16, Proposition 2.7]. With this at hand the proof follows the same lines as the proof of Theorem 1.1 by using Proposition 6.2 and $\sigma(H)=\sigma\left(H_{\varphi}\right)$.

\section{Acknowledgments}

The authors wish to thank Daniel Lenz and Marcel Schmidt for pointing out the papers [BdMLS09, LSV09, LSV11]. They acknowledge the support of the Israel Science Foundation (grants No. 970/15) founded by the Israel Academy of Sciences and Humanities.

\section{REFERENCES}

[Agm83] S. Agmon, On positivity and decay of solutions of second order elliptic equations on Riemannian manifolds, 19-52, Methods of functional analysis and theory of elliptic equations (Naples, 1982), Liguori, Naples, 1983.

[BM95] M. Biroli and U. Mosco, A Saint-Venant type principle for Dirichlet forms on discontinuous media, Ann. Mat. Pura Appl. (4) 169 (1995), 125-181.

[BH91] N. Bouleau and F. Hirsch, Dirichlet forms and analysis on Wiener space, De Gruyter Studies in Mathematics, vol. 14, Walter de Gruyter \& Co., Berlin, 1991.

[BdMLS09] A. Boutet de Monvel, D. Lenz, and P. Stollmann, Sch'nol's theorem for strongly local forms, Israel J. Math. 173 (2009), 189-211.

[BdMS03] A. Boutet de Monvel and P. Stollmann, Eigenfunction expansions for generators of Dirichlet forms, J. Reine Angew. Math. 561 (2003), 131-144.

[CFKS87] H. L. Cycon, R. G. Froese, W. Kirsch, and B. Simon, Schrödinger operators with application to quantum mechanics and global geometry, study ed., Texts and Monographs in Physics, SpringerVerlag, Berlin, 1987. 
[Dav95] E. B. Davies, Spectral theory and differential operators, Cambridge Studies in Advanced Mathematics, vol. 42, Cambridge University Press, Cambridge, 1995.

[DFP14] B. Devyver, M. Fraas, and Y. Pinchover, Optimal Hardy weight for second-order elliptic operator: an answer to a problem of Agmon, J. Funct. Anal., 266 (2014), 4422-4489.

[FLW14] R. L. Frank, D. Lenz, and D. Wingert, Intrinsic metrics for non-local symmetric Dirichlet forms and applications to spectral theory, J. Funct. Anal. 266 (2014), 4765-4808.

[Fuk80] M. Fukushima, Dirichlet forms and Markov processes, North-Holland Mathematical Library, vol. 23, North-Holland Publishing Co., Amsterdam-New York; Kodansha, Ltd., Tokyo, 1980.

[FOT94] M. Fukushima, Y. Ōshima, and M. Takeda, Dirichlet forms and symmetric Markov processes, De Gruyter Studies in Mathematics, vol. 19, Walter de Gruyter \& Co., Berlin, 1994.

[HK11] S. Haeseler, and M. Keller, Generalized solutions and spectrum for Dirichlet forms on graphs. Random walks, boundaries and spectra, Progr. Probab., 64, Birkhäuser/Springer Basel AG, Basel (2011), 181-199.

[HKM93] J. Heinonen, T. Kilpeläinen, and O. Martio, Nonlinear potential theory of degenerate elliptic equations, Oxford Mathematical Monographs, The Clarendon Press, Oxford University Press, New York, Oxford Science Publications, 1993.

[HKMW13] X. Huang, M. Keller, J. Masmune, and R. K. Wojciechowski, A note on self-adjoint extensions of the Laplacian on weighted graphs, J. Funct. Anal. 265 (2013), 1556-1578.

[KL12] M. Keller, and D. H. Lenz, Dirichlet forms and stochastic completeness of graphs and subgraphs, J. Reine Angew. Math. 666 (2012), 189-223.

[KPP16] M. Keller, Y. Pinchover, and F. Pogorzelski, Optimal hardy inequalities for schrödinger operators on graphs, arXiv:1612.04051, 2016.

[LSV09] D. H. Lenz, P. Stollmann, and I. Veselić, The Allegretto-Piepenbrink theorem for strongly local forms, Documenta Mathematica 14 (2009), 167-189.

[LSV11] D. H. Lenz, P. Stollmann, and I. Veselić, Generalized eigenfunctions and spectral theory for strongly local Dirichlet forms, Oper. Theory Adv. Appl. 214 (2011), 83-106.

[MR92] Z. M. Ma and M. Röckner, Introduction to the theory of (nonsymmetric) Dirichlet forms, Universitext, Springer-Verlag, Berlin, 1992.

[P88] Y. Pinchover, On positive solutions of second-order elliptic equations, stability results and classifcation, Duke Math. J. 57 (1988), 955-980.

[P07] Y. Pinchover, Topics in the theory of positive solutions of second-order elliptic and parabolic partial differential equations, in "Spectral Theory and Mathematical Physics: A Festschrift in Honor of Barry Simon's 60th Birthday", eds. F. Gesztesy, et al., 329-356, Proceedings of Symposia in Pure Mathematics 76, American Mathematical Society, Providence, RI, 2007.

[P07a] Y. Pinchover, A Liouville-type theorem for Schrödinger operators, Comm. Math. Phys. 272 (2007), 75-84.

[PT06] Y. Pinchover and K. Tintarev, A ground state alternative for singular Schrödinger operators, J. Funct. Anal., 230 (2006), 65-77.

[Pin95] R. G. Pinsky, Positive harmonic functions and diffusion, Cambridge Studies in Advanced Mathematics, vol. 45, Cambridge University Press, Cambridge, 1995.

[Shn57] È. È. Šhnol', On the behavior of the eigenfunctions of Schrödinger's equation, Mat. Sb. (N.S.), 42, (84) (1957), 273-286; erratum, 46 (88) (1957), 259.

[Shu92] M. .A. Shubin, Spectral theory of elliptic operators on noncompact manifolds, 35-108, Méthodes semi-classiques, Vol. 1 (Nantes, 1991), Astérisque, vol. 207, 1992.

[Sim81] B. Simon, Spectrum and continuum eigenfunctions of Schrödinger operators, J. Funct. Anal., 42 (1981), 347-355.

[Sto01] P. Stollmann, Caught by disorder, Bound states in random media, Progress in Mathematical Physics, vol. 20, Birkhäuser, Boston, Inc., Boston, MA, 2001.

Department of Mathematics, Technion - Israel Institute of Technology, Haifa, Israel

E-mail address: beckus.siegf@technion.ac.il

Department of Mathematics, Technion - Israel Institute of Technology, Haifa, Israel

E-mail address: pincho@technion.ac.il 